\title{
Neurogranin and BACE1 in CSF as Potential Biomarkers Differentiating Depression with Cognitive Deficits from Early Alzheimer's Disease: A Pilot Study
}

\author{
Carola G. Schipke $^{a} \quad$ Ann De Vos ${ }^{b}$ Manuel Fuentes ${ }^{c}$ Dirk Jacobs ${ }^{a}$ \\ Eugeen Vanmechelen ${ }^{a}$ Oliver Peters ${ }^{c, d}$ \\ ${ }^{a}$ Charité - Universitätsmedizin Berlin, corporate member of Freie Universität Berlin, \\ Humboldt-Universität zu Berlin, and Berlin Institute of Health (BIH), Neuropathology, \\ Berlin, Germany; ${ }^{b}$ ADx NeuroSciences NV, Gent, Belgium; ${ }^{c}$ Charité - Universitätsmedizin \\ Berlin, corporate member of Freie Universität Berlin, Humboldt-Universität zu Berlin, \\ and Berlin Institute of Health (BIH) and German Center for Neurodegenerative Diseases \\ (DZNE), Department of Psychiatry and Psychotherapy, Berlin, Germany; ${ }^{\mathrm{d}}$ German Center for \\ Neurodegenerative Diseases (DZNE), Berlin, Germany
}

Keywords

Neurogranin · BACE1 protein · Cerebrospinal fluid · Alzheimer's disease · Depression · ELISA

\begin{abstract}
Background/Aims: Major depressive disorder (MDD) can cooccur with early Alzheimer's disease (AD) or may cause memory problems independently of AD. Previous studies have suggested that the AD-related cerebrospinal fluid (CSF) biomarkers tau and $A \beta(1-42)$ could help discriminate between early $A D$ and depression unrelated to AD. Moreover, the postsynaptic protein neurogranin and presynaptic BACE1 have increasingly gained attention as potential new AD biomarkers, but they have not yet been investigated concerning depression. Methods: Using ELISAs, we studied CSF neurogranin and BACE1 levels in patients with mild $(n=21)$ and moderate $(n=19) \mathrm{AD}$, as well as in MDD patients with $(n=20)$ and without $(n=20)$ cognitive deficits. The clinical examinations included analyses of $t$-tau, $A \beta(1-42)$, and $A \beta(1-40)$, besides neuropsychological tests and cranial magnetic resonance imaging. Depressive symptom severity was assessed using the Geriatric Depression Scale (GDS). Results: Along with
\end{abstract}

Carola G. Schipke and Ann De Vos contributed equally to this study. 
classic AD biomarkers, neurogranin and BACE1 CSF levels differed between moderate AD and MDD $(p \leq 0.01)$. MDD associated with cognitive deficits was distinguished from mild AD through the CSF neurogranin/BACE1 ratio $(p<0.05)$, which was strongly correlated with GDS scores ( $\rho=-0.656 ; p<0.01)$. Conclusion: The neurogranin/BACE1 ratio in CSF can distinguish between depression and $A D$ among patients with similar cognitive deficits, along with the classic AD biomarkers. Further longitudinal studies are ongoing to identify which biomarkers have prognostic value.

(C) 2018 The Author(s)

Published by S. Karger AG, Basel

\section{Introduction}

A major goal in current clinical research on Alzheimer's disease (AD) is to identify the disease in its earliest stages. Memory clinic patients exhibiting cognitive deficits may in fact have early symptoms of $\mathrm{AD}$, but similar deficits are found in individuals with major depressive disorder (MDD). The clinical appearance of numerous patients is characterized by symptoms that may belong to AD, MDD, or both. There are hints that the cognitive decline found in the two disorders may at least partially share an underlying pathophysiology: when the neuropathological hallmarks of AD - i.e., amyloid plaques and intracellular tau tangles - spread from the entorhinal cortex into the limbic system, patients are prone to develop anxiety and depressive symptoms. Moreover, MDD has been discussed as a risk factor for the development of $\mathrm{AD}[1,2]$. Essentially, discriminating between AD and MDD is a prerequisite for an optimal clinical and pharmacological treatment [3].

Many studies have employed neuropsychological test profiles to discriminate between $\mathrm{AD}$ and MDD [1, 2, 4, 5], yielding differences between healthy controls, patients with mild cognitive impairment (MCI) due to $\mathrm{AD}$, and $\mathrm{AD}$ patients. However, this approach is less successful in distinguishing patients with early stages of AD from MDD patients with cognitive deficits or other disorders [2].

There are reports suggesting that AD-related biomarkers in cerebrospinal fluid (CSF) could distinguish the presence of AD pathology from depression. Kramberger et al. [6] examined patients' CSF to determine whether comorbid depressive symptoms have an effect on specific biomarkers associated with AD. They measured CSF levels of total tau protein ( $t$-tau) and amyloid beta 1-42 protein $(A \beta(1-42))$ in depressed patients suffering from cooccurring subjective cognitive impairment or $\mathrm{AD}$, and found higher CSF t-tau levels in $\mathrm{AD}$ patients, while there was no strong association between t-tau or $A \beta(1-42)$ levels and depressive symptoms. These findings, suggesting that the biomarkers only represent the neuronal degeneration typical of $\mathrm{AD}$, were corroborated by another report where increased CSF t-tau levels were noted in MCI due to AD and in AD compared to depression. In contrast, decreased t-tau levels were found in depressed patients compared to a control group [7], while there were no differences in CSF $A \beta(1-42)$ levels between depressed patients and those deemed cognitively healthy. A difference was only found when contrasting depression with $\mathrm{MCI}$ due to AD and with AD [7]. Yet, such findings are debated, since other explorative studies did not agree on all aspects. While unaltered CSF t-tau levels comparing controls and MDD patients have been confirmed in an independent study [8], the CSF A $\beta(1-42)$ levels in this study were decreased in MDD patients compared to cognitively healthy persons, whereas another study again demonstrated higher CSF A $\beta(1-42)$ levels in MDD patients [9]. Taken together, CSF biomarkers may have a role in differentiating early AD from depression with cognitive impairment, but more studies are needed to clarify their exact role. Additional biomarkers, reflecting differences in neuropathological processes, need to be considered as well when further exploring the distinctive CSF biomarker profiles of AD and MDD. 
Several recent studies have indicated that a slow but progressive loss of synapses is one of the earliest events in $\mathrm{AD}$, likely directly linked to cognitive symptoms. This can indirectly be observed by measuring levels of the postsynaptic protein neurogranin in brain tissue [1012]. Decreased levels of neurogranin, a protein that has a key role in long-term potentiation and learning [13-15], have been found in brains of AD patients [10,16], while CSF neurogranin levels are increased [17-20]. Studies have suggested that increased CSF neurogranin levels might even be predictive of progression from MCI to AD; thus, this protein has also been discussed as a potential AD biomarker [21,22]. Moreover, studies have proposed that neurogranin might be an AD-specific biomarker within the spectrum of neurodegenerative diseases, since no substantial changes in neurogranin levels were observed in other forms of neurodegeneration accompanied with cognitive impairment $[23,24]$. Determining CSF beta-secretase 1 (BACE1) levels, a predominantly presynaptic protein that initiates the formation of the aggregation-prone $\mathrm{A} \beta(1-42)$ in brains with $\mathrm{AD}[25,26]$, might also be useful as a prognostic biomarker based on a recent finding. That is, the ratio of neurogranin to BACE1 in CSF is correlated with cognitive decline in both $\mathrm{MCI}$ and $\mathrm{AD}$ [27]. To our knowledge, this is the first investigation of BACE1 in MDD. Up to now, AD studies have presented conflicting results, ranging from increased [28] to unchanged CSF levels [27, 29, 30].

In depression, synaptic loss may play a role as well $[31,32]$. Changes in neurogranin levels have been reported to occur in the brain [31] and in CSF [33]. Interestingly, the study reporting the latter result found lower CSF neurogranin levels in depressed patients than in healthy controls. While the findings did not reach a level of statistical significance, probably due to the low number of patients, they suggest altered neurogranin levels as a potential biomarker distinguishing healthy controls and MDD patients from AD patients.

In the present study, we explored the CSF levels of neurogranin and BACE1 in MDD patients with and without cognitive deficits, and compared them to the CSF levels in patients suffering from mild and moderate dementia due to AD.

\section{Subjects and Methods}

\section{Study Population and Clinical and Neuropsychometric Assessments}

The patients included in this study are part of a local cohort at the Memory Clinic of Charité - Universitätsmedizin Berlin. They were examined and diagnosed according to the Diagnostic Criteria for Major Depressive Disorder and Depressive Episodes (DSM-5 criteria). Physicians and neuropsychologists carried out a comprehensive clinical workup of each patient, including a medical history, psychiatric and neurological examination, neuropsychological testing to evaluate cognitive and functional performance, a lumbar puncture, and cranial magnetic resonance imaging (cMRI). In this study, we focused only on patients with a diagnosis of MDD and mild or moderate neurocognitive disorders attributed to AD. To exclude overlapping disease patterns and the contribution of other neuropathological processes, patients who met the DSM-5 criteria for both MDD and AD or other neuropsychiatric diseases were excluded.

To obtain a clinical diagnosis of AD or MDD, trained and certified neuropsychologists performed the following neuropsychological examinations. We administered a neuropsychological test battery established by the Consortium to Establish a Registry for Alzheimer's Disease (CERAD). The CERAD battery is a highly standardized neuropsychological tool to assess various stages of cognitive deficit in patients with suspected AD [34, 35]. Additionally, all patients were administered the Trail Making Tests A and B, as well as the clock drawing test and the revised version of the Wechsler Memory Test (CERAD-Plus). Functional decline was assessed by the B-ADL, a questionnaire to be completed by the primary caregiver or a 
family member known to the patient. For our analyses, all the results of the neuropsychological scales were $z$-standardized with regard to sex, age, and years of education. Depressive symptoms were quantified using the original version of the Geriatric Depression Scale (GDS) [36]. The GDS is a manageable, self-administered questionnaire that has been shown to deliver a valid assessment of depression in elderly subjects [37]. In 4 clinically clear cases of MDD according to the DSM-5, the GDS was not administered. Similarly, 1 patient was diagnosed as not being depressed without undergoing a GDS assessment.

CSF was collected and analyzed according to strictly standardized protocols described elsewhere [38]. Briefly, this involved collecting $12 \mathrm{~mL}$ of CSF in polypropylene tubes. Immediately after collection, the tubes were gently shaken and centrifuged $(1,600 \mathrm{~g}$; room temperature; $10 \mathrm{~min})$, aliquoted $(500 \mu \mathrm{L})$, and frozen within $30 \mathrm{~min}$. The material was stored at $-80^{\circ} \mathrm{C}$. To quantify amyloid peptides, we used the V-PLEX A $\beta$ Peptide Panel 1 (6E10) Kit (Meso Scale Diagnostics, Rockville, MD, USA). For t-tau, we used the INNOTEST ${ }^{\circledR}$ hTAU Ag (Fujirebio Germany GmbH, Hannover, Germany). Under these conditions, the following CSF biomarker values were rated as indicative of $A D$ : $A \beta(1-42)<600 \mathrm{pg} / \mathrm{mL}$ or $A \beta(1-42) /$ $\mathrm{A} \beta(1-40)$ ratio $\leq 0.065$, in addition to t-tau $>350 \mathrm{pg} / \mathrm{mL}$. The patient CSF included in this study was largely selected to match these CSF diagnostic criteria in order to obtain a cohort of neurobiologically validated AD patients.

Diagnoses were made at a consensus conference composed of psychiatrists, neurobiologists, and neuropsychologists according to the DSM-5. The patients' history, the standard cognitive and functional measurements, the CSF biomarker values for t-tau and amyloid peptides, and the cMRI findings were taken into account for this purpose. The cMRI findings likewise helped to exclude extensive vascular encephalopathy and to quantify brain atrophy. A diagnosis of MDD with cognitive deficits ( $\operatorname{Cog} D$ ) was made if the CERAD test battery revealed at least one cognitive domain to be significantly below average ( $Z$-score $\leq-1.5$ ), if no substantial problems were indicated on the B-ADL, and if no neurobiological signs of AD in CSF and/or brain atrophy were found. This procedure resulted in the formation of the following four diagnostic groups: (1) MDD with $\operatorname{Cog} D(M D D / \operatorname{Cog} D)$, (2) MDD without $\operatorname{Cog} D(M D D / \operatorname{Cog} N)$, (3) mild neurocognitive disorders due to AD (ADmild), and (4) moderate neurocognitive disorders due to AD (ADmod), according to the DSM-5 criteria and CERAD-Plus (Table 1). The MDD/CogD and ADmild groups did not differ in MMSE scores or other cognitive measures, while the MDD/CogN and MDD/CogD groups did not differ in GDS scores.

\section{CSF Biomarker Assessments}

CSF neurogranin and BACE1 levels were measured using ELISA, as previously described [27]. In short, the neurogranin assay involved two monoclonal antibodies -ADx403 (clone ADxNGCI2) and ADx451 (clone ADxNGCT1) - and quantified the amount of neurogranin in 15 $\mu \mathrm{L}$ of undiluted sample during a 3-h incubation protocol. Final concentrations of CSF neurogranin were intrapolated $\left(\log _{x} ; 5 \mathrm{PL}\right)$ using a synthetic calibrator. Intra-assay variability between duplicates of CSF samples $(n=80)$ (coefficient of variation, $\% \mathrm{CV}$ ) in this study was $5 \% \mathrm{CV}$. Subsequent to the first analysis, neurogranin levels were remeasured in 2 samples in which the optic density value surpassed the upper limit of the assay. A 4-fold dilution was used during the second run, which was corrected after intrapolation. The BACE1 ELISA is commercialized by Euroimmun AG (Lübeck, Germany) and involved lyophilized, ready-to-use calibrators and a standardized protocol that was harmonized with the procedures used for the other AD biomarkers. BACE1 levels were measured according to the kit insert, where concentrations were calculated via intrapolation (5PL curve fit; $\log _{\mathrm{X}}$ ) based on the calibrator curve. Intra-assay precision in this study was $3 \% \mathrm{CV}$, based on duplicate measurements of the CSF samples.

CSF levels of t-tau, $A \beta(1-42)$, and $A \beta(1-40)$ were redetermined for this study using ELISAs by Euroimmun (Table 1; online suppl. material; for all online suppl. material, see 
Schipke et al.: Neurogranin and BACE1 in CSF as Differential Diagnostic Biomarkers

Table 1. Summary of the demographic, clinical, and biochemical data for the diagnostic groups

\begin{tabular}{|c|c|c|c|c|}
\hline & $\mathrm{MDD} / \operatorname{CogN}$ & $\mathrm{MDD} / \operatorname{Cog} \mathrm{D}$ & ADmild & ADmod \\
\hline \multicolumn{5}{|l|}{ Demographic data } \\
\hline $\operatorname{Sex}(\mathrm{F} / \mathrm{M}), n$ & $20(10 / 10)$ & $20(14 / 6)$ & $21(13 / 8)$ & $19(11 / 8)$ \\
\hline Age at LP, years & $64(44-83)$ & $67(46-83)$ & $74(64-86)$ & $71(59-89)$ \\
\hline \multicolumn{5}{|l|}{ Clinical data } \\
\hline GDS score & $18(6-22)$ & $17(11-20)$ & $7(5-11.5)$ & $12(5-15)$ \\
\hline MMSE score at LP $(/ 30)$ & $29(27-30)$ & $26(18-30)$ & $27(25-29)$ & $17(14-20)$ \\
\hline Verbal Fluency score & $22(18-27)$ & $20(15-22)$ & $15(10-21.75)$ & $9(6.75-11.25)$ \\
\hline Boston Naming Test score (/15) & $15(14-15)$ & $15(13-15)$ & $14(12.5-14.5)$ & $13(8-14)$ \\
\hline Word List Delayed Recall score (/10) & $7(6-9)$ & $5(3-7)$ & $3(2-4)$ & $1(0-2)$ \\
\hline Constructional Praxis Delayed Recall score (/11) & $11(8-11)$ & $8(6-9)$ & $4(1.5-6.5)$ & $2(0-5)$ \\
\hline Clock drawing test score (1-6) & $1(1-1.75)$ & $2(2-3)$ & $2(1-3)$ & $4(3-5)$ \\
\hline Trail Making Test A score (/180) & $43(23.75-53.5)$ & $44(41-61)$ & $59(39-90.5)$ & $124(77-180)$ \\
\hline Trail Making Test B score (/300) & $85.5(57-118.5)$ & $147.5(109.3-252.8)$ & $224(111-300)$ & $300(300-300)$ \\
\hline \multicolumn{5}{|l|}{ Biochemical data } \\
\hline CSF total-tau, pg/mL & $307(276-358)$ & $384(267-559)$ & $651(508-676)$ & $699(633-951)$ \\
\hline $\operatorname{CSF} A \beta(1-42), \mathrm{pg} / \mathrm{mL}^{1}$ & $601(503-734)$ & $455(380-597)$ & $340(232-418)$ & $325(257-355)$ \\
\hline CSF A $\beta(1-40), \mathrm{pg} / \mathrm{mL}$ & $6,474(5,202-7,390)$ & $5,836(4,915-8,103)$ & $6,274(4,601-8,029)$ & $8,005(5,912-9,018)$ \\
\hline $\operatorname{CSF} A \beta(1-42) / A \beta(1-40)$ & $0.098(0.089-0.112)$ & $0.077(0.058-0.101)$ & $0.053(0.044-0.067)$ & $0.039(0.033-0.043)$ \\
\hline CSF A $\beta(1-42) /$ total-tau & $2.065(1.605-2.409)$ & $1.325(0.748-1.933)$ & $0.528(0.377-0.716)$ & $0.392(0.295-0.526)$ \\
\hline
\end{tabular}

Clinical variables are summarized as median values with the 25th and 75th quartiles in parentheses. Maximum scores are given in parentheses as "/n." ADmild, mild Alzheimer's disease; ADmod, moderate Alzheimer's disease; MDD/CogN, depression without cognitive deficits; MDD/CogD, depression with cognitive deficits; GDS, Geriatric Depression Scale; LP, lumbar puncture; MMSE, Mini-Mental State Examination. ${ }^{1}$ The levels of 1 sample, in the ADmod group, were below the lower limit of quantification and were excluded from analysis.

www.karger.com/doi/10.1159/000489847) to eliminate preanalytical differences in quantification, as compared to the neurogranin and BACE1 analyses. All assays included lyophilized, ready-to-use calibrators and standardized protocols and were performed according to the kit insert. Based on duplicates of the CSF samples, the intra-assay $\% \mathrm{CV}$ were: $5 \% \mathrm{CV}$ for t-tau, $5 \% \mathrm{CV}$ for $\mathrm{A} \beta(1-42)$, and $4 \% \mathrm{CV}$ for $\mathrm{A} \beta(1-40)$. The ELISAs for all analytes were run blinded from the clinical diagnosis. Another sample, also from the ADmod group, contained levels of $A \beta(1-42)$ that lay below the lower limit of quantification, resulting in this patient being excluded from the statistical analyses. All other samples could be quantified within the dynamic range of the respective ELISAs.

\section{Statistical Analyses}

GraphPad Prism 6.02 was used for the statistical analyses and figures. To compare groups, a Kruskal-Wallis test was applied to the quantitative data, as they were nonnormally distributed. Normality was checked by a D'Agostino-Pearson normality test and a ShapiroWilk normality analysis. The strength of the correlation between analytes was investigated using Spearman's correlation testing. MedCalc (version 16.1.2) was used to obtain a nonparametric estimate of the area under the curve (AUC) in the ROC (receiver operating characteristic) analyses. Results were considered significant for $p$ values $<0.05$.

\section{Results}

\section{Clinical Comparisons of the Four Diagnostic Groups}

We analyzed four groups: MDD patients with or without $\operatorname{Cog}$, as well as patients exhibiting mild or moderate AD. Table 1 summarizes the demographic and descriptive data for the cohort. Statistical analysis confirmed that there were no differences in MMSE scores or the other cognitive measures between MDD/CogD and ADmild as determined by CERAD-Plus. 
Fig. 1. Scatter dot plots demonstrating CSF concentrations of neurogranin (a) and BACE1 (b) and the neurogranin/BACE1 ratio in CSF (c). Median levels are depicted as lines in each plot; bars represent the interquartile range. Statistically significant differences: ${ }^{*} p<0.05,{ }^{* *} p<0.01,{ }^{* * *} p<$ $0.001, * * * * p<0.0001$. MDD/ CogN, depression without cognitive deficits; MDD/CogD, depression with cognitive deficits; ADmild, mild Alzheimer's disease; ADmod, moderate Alzheimer's disease.
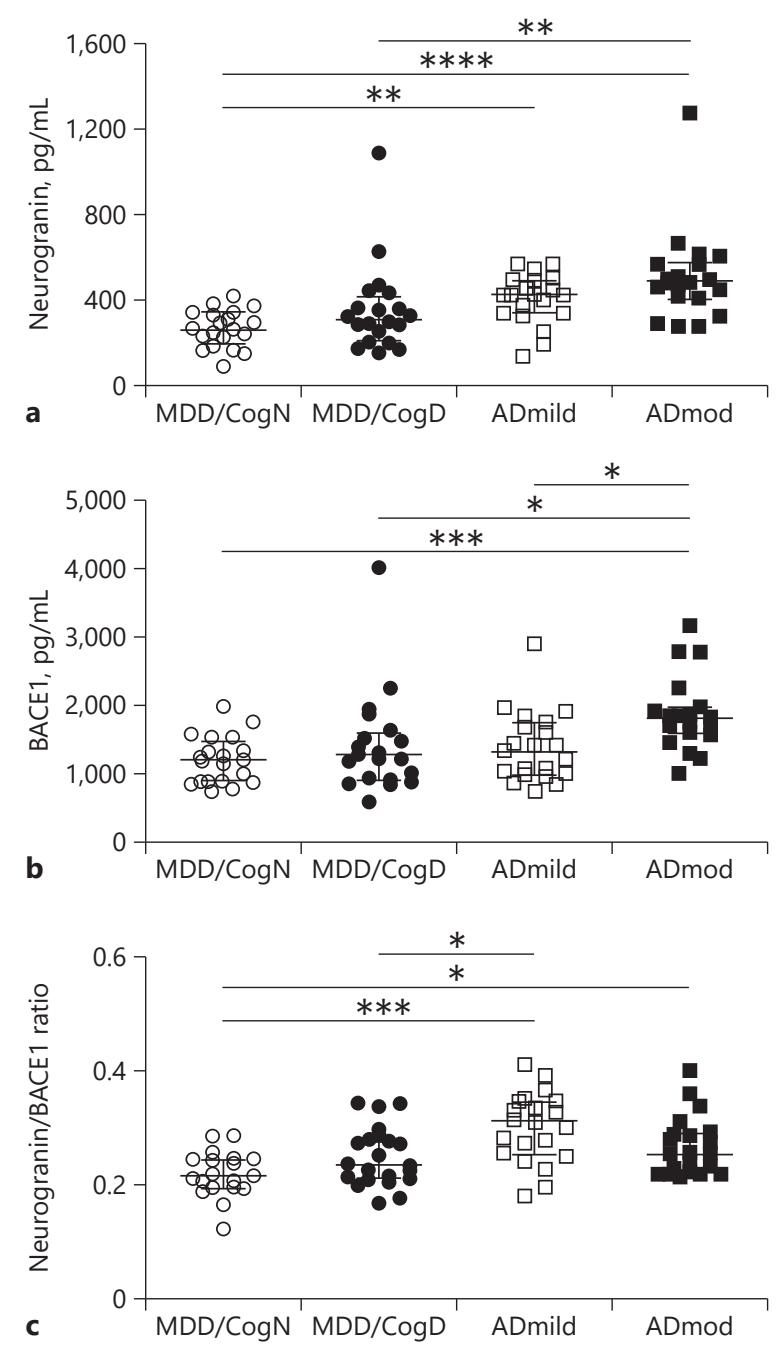

Regarding depression severity, as quantified by the GDS, no differences between MDD/CogN and MDD/CogD were found. Slightly elevated GDS scores were noted in ADmod compared to ADmild, most likely because in more advanced stages of the disease, AD patients may develop symptoms resembling depression, or it may be more difficult to obtain reliable GDS scores.

\section{Synaptic CSF Biomarkers in Depression versus AD}

As shown in Figure 1, quantifications of the synaptic proteins neurogranin and BACE1 in CSF revealed substantial differences between MDD and AD. Significant differences in both synaptic biomarkers were determined between MDD/CogN and ADmod (neurogranin, $p<$ 0.0001; BACE1, $p<0.001$; Fig. 1). MDD/CogN and ADmild differed significantly in terms of CSF neurogranin levels $(p<0.01)$. Furthermore, neurogranin $(p<0.01)$ and BACE1 $(p<0.05)$ levels were increased in ADmod versus MDD/CogD. No significant differences in either analyte were found in the comparison of $\mathrm{MDD} / \operatorname{Cog} N$ with $M D D / \operatorname{Cog} D$, while mild and moderate AD differed significantly only in BACE1 levels $(p<0.05$; Fig. 1$)$. Neurogranin or BACE1 alone did not differ significantly in the comparison of MDD/CogD with ADmild. 
Table 2. ROC analysis of the single analytes and their ratios
AUC values for MDD/

CogD vs. ADmild

$\begin{array}{ll}\text { Classic } & \\ \text { Tau } & 0.805^{* * * *} \\ \text { A } \beta(1-42) & 0.748^{* * * *} \\ \text { Novel } & 0.696^{*} \\ \text { Neurogranin } & 0.523 \\ \text { BACE1 } & 0.742^{* * *} \\ \text { Neurogranin/BACE1 ratio } & \\ \text { Combinations } & 0.727^{* * *} \\ \text { Neurogranin/tau } & 0.814^{* * * *} \\ \text { Neurogranin/A }(1-42) & 0.818^{* * * *} \\ \text { BACE1/tau } & 0.769^{* * *} \\ \text { BACE1/A } \beta(1-42) & \end{array}$

Area under the curve (AUC) values from the receiver operating characteristic (ROC) analyses; log-transformed data were used for calculations due to the nonnormality of the data. Statistically significant differences: $* p<0.05$, *** $p<0.001$, **** $p<0.0001$. ADmild, patients with mild Alzheimer's disease; MDD/CogD, major depressive disorder with cognitive deficits.

We next assessed the neurogranin/BACE1 ratio in all groups, and detected a significant difference between MDD/CogD and ADmild $(p<0.05)$ comparable to that found for t-tau (online suppl. material; $p<0.05$ ). When the results of the replicated measurements of the well-characterized $\mathrm{AD}$ biomarkers tau and $\mathrm{A} \beta$ were analyzed, the results were in line with the clinical diagnoses, as expected. Levels of t-tau were substantially higher in the ADmod group than in the MDD/CogN group ( $p<0.0001)$, whereas $A \beta(1-42)$ concentrations were lower $(p<0.0001)$. Furthermore, the $A \beta(1-42) / A \beta(1-40)$ ratio and also the $A \beta(1-42) /$ tau ratio differed between the MDD/CogN group and both the groups of ADmild and ADmod patients. Among MDD patients, CSF $A \beta(1-42)$ and the $A \beta(1-42) /$ tau ratio distinguished patients with from those without cognitive deficits.

\section{Discriminating Power of CSF Biomarkers to Distinguish between Cognitive Deficits due to $M D D$ and Cognitive Deficits due to $A D$}

We next performed a ROC analysis. Since this was an exploratory study with a limited number of samples per group, the resulting AUC values should be carefully interpreted. However, the results offer a preliminary indication of the potential of the analytes in discriminating between MDD and AD. Table 2 presents the AUC values for the single biomarkers and combinations for the distinction of MDD/CogD from ADmild. As described in the Subjects and Methods section, the selection criteria for CSF samples included t-tau and $A \beta(1-42)$ values. Thus, the AUC values were significantly high when comparing MDD/CogD with ADmild in case of t-tau (AUC 0.805; $p<0.0001$ ) as well as A $\beta(1-42$ ) (AUC $0.748 ; p<0.0001$ ). Interestingly, the AUC value for neurogranin was rather moderate as a single analyte (AUC 0.696; $p<$ 0.050), while BACE1 had no diagnostic value on its own (AUC 0.523; ns). Yet, when combined, the neurogranin/BACE1 ratio became more informative (AUC $0.742 ; p<0.001$ ). Testing multiple combinations of biomarkers, the highest power of discrimination between MDD/ $\operatorname{Cog} \mathrm{D}$ and ADmild was found for the ratios of neurogranin/A $\beta(1-42)$ (AUC $0.814 ; p<0.0001$ ) and BACE1/tau (AUC 0.818; $p<0.0001$ ). 
Table 3. Correlation analysis between GDS scores and CSF analytes in all groups combined

\begin{tabular}{lcc}
\hline & $p$ value & $p$ value \\
\hline Single analyte & & \\
$\quad$ Neurogranin & $\mathbf{- 0 . 2 7 8}$ & $\mathbf{0 . 0 1 5}$ \\
BACE1 & -0.100 & 0.391 \\
Tau & $\mathbf{- 0 . 3 3 3}$ & $\mathbf{0 . 0 0 3}$ \\
A $\beta(1-42)$ & $\mathbf{0 . 2 4 4}$ & $\mathbf{0 . 0 3 4}$ \\
A $\beta(1-40)$ & -0.091 & 0.435 \\
Ratio of analytes & & \\
Neurogranin/BACE1 & $\mathbf{- 0 . 3 3 0}$ & $\mathbf{0 . 0 0 4}$ \\
Neurogranin/tau & 0.138 & 0.235 \\
Neurogranin/A $\beta(1-42)$ & $\mathbf{- 0 . 3 1 6}$ & $\mathbf{0 . 0 0 6}$ \\
BACE1/tau & $\mathbf{0 . 3 0 4}$ & $\mathbf{0 . 0 0 8}$ \\
BACE1/A $\beta(1-42)$ & $\mathbf{- 0 . 2 6 8}$ & $\mathbf{0 . 0 2 0}$ \\
\hline
\end{tabular}

Spearman's correlation coefficient values are presented, with the corresponding statistical $p$ values. Statistically significant values are marked in bold font. GDS, Geriatric Depression Scale.

Table 4. Correlation matrix of CSF analytes in all groups combined

\begin{tabular}{cccccc}
\hline & Neurogranin & BACE1 & Total tau & A $\beta(1-42)$ & A $\beta(1-40)$ \\
\hline Neurogranin & - & & & & \\
$p$ value & - & & & & \\
BACE1 & 0.853 & - & & & \\
$p$ value & $<0.0001$ & - & & & \\
Total tau & 0.896 & 0.733 & - & & \\
$p$ value & $<0.0001$ & $<0.0001$ & - & & \\
A $\beta(1-42)$ & -0.197 & 0.043 & -0.412 & - & - \\
$p$ value & ns & ns & 0.001 & - & - \\
A $3(1-40)$ & 0.642 & 0.700 & 0.484 & 0.388 & $<001$ \\
$p$ value & $<0.0001$ & $<0.0001$ & $<0.0001$ & $<0.001$ & - \\
\hline
\end{tabular}

Spearman's correlation coefficients are presented, with the corresponding statistical $p$ values.

\section{Correlation between GDS Scores and CSF Analytes}

We additionally addressed the question of whether it might be possible to link depression severity to quantitative levels of specific biomarker proteins. Therefore, we performed correlation analyses on the whole study cohort using GDS scores. GDS scores correlated weakly with levels of neurogranin, tau, and $A \beta(1-42)$, while no correlation was found with BACE1 or $A \beta(1-40)$ (Table 3). The highest correlation coefficient was found between GDS scores and the neurogranin/BACE1 ratio. In separate group analyses, significant correlations could be established for the group MDD/CogD between GDS scores and the neurogranin/BACE1 ratio $(\rho=-0.656 ; p<0.01)$, the neurogranin $/ \mathrm{A} \beta(1-42)$ ratio $(\rho=-0.467 ; p<0.05)$, the BACE1 $/$ tau ratio $(\rho=0.568 ; p<0.05)$, and tau $(\rho=-0.520 ; p<0.05)$.

\section{Correlation between Different CSF Biomarkers}

Table 4 gives an overview of the relationship between CSF analytes expressed as Spearman's correlation coefficients. Overall, neurogranin, BACE1, and t-tau exhibited the strongest correlations (Table 4). 
$\mathrm{A} \beta(1-42)$ and t-tau were moderately associated $(\rho=-0.412 ; p<0.001)$, while there was no relationship between levels of A $\beta(1-42)$ and neurogranin $(\rho=-0.197$; ns) or BACE1 ( $\rho=$ 0.043 ; ns). Similarly, there was a weak association between $A \beta(1-42)$ and $A \beta(1-40)(\rho=$ $0.388 ; p<0.001$ ).

\section{Discussion}

In the present exploratory study, we analyzed the CSF levels of the synaptic proteins neurogranin and BACE1 in case of AD and MDD. In addition to the classic AD biomarkers tau, $A \beta(1-42)$, and $A \beta(1-40)$, we analyzed their values in the CSF of previously characterized AD patients and MDD patients. We found that, like protein tau and $A \beta(1-42)$, the ratio of neurogranin/BACE1 might discriminate between these different cognitive impairment etiologies, even if the cognitive abilities are similar, as expressed in MMSE scores. Among the investigated biomarkers, the neurogranin/BACE1 ratio also showed the strongest correlation with depression severity in the overall cohort. Specifically, in MDD patients with cognitive deficits, high neurogranin/BACE1 CSF levels were associated with less severe depressive symptoms, possibly indicating that these patients are at risk of future development of AD.

CSF levels of $A \beta(1-42)$ and t-tau are well-characterized AD biomarkers. They are linked to the disruption of amyloid metabolism and plaque pathology, and to the cortical neurodegeneration occurring in the disease [39]. Since levels of neurogranin are increased in the CSF of $\mathrm{AD}$ patients, while they are decreased in AD brains, this protein has also been discussed as a potential AD biomarker [17-22]. Moreover, studies have suggested that neurogranin might be an AD-specific biomarker. No significant changes in neurogranin levels were found in other neurodegenerative disorders [23, 24]. To the best of our knowledge, this is the first investigation of BACE1 in MDD. Regarding AD, on the other hand, there are conflicting reports, suggesting that CSF levels are increased [28] or unchanged [27, 29, 30]. As presented here, our results corroborate an $\mathrm{AD}$-specific increase in CSF levels of neurogranin. The patients suffering from moderate AD exhibited significantly elevated neurogranin CSF levels when compared to the MDD patients without any cognitive symptoms. The inclusion of increased CSF levels of $\mathrm{t}$-tau in the identification of $\mathrm{AD}$ patients, combined with the strong correlation observed between neurogranin and protein tau, may explain this finding. When combined with BACE1, the ratio of neurogranin/BACE1 even discriminated between patients with mild $\mathrm{AD}$ and depressed patients with a similar level of cognitive impairment.

In patients suffering from MDD with cognitive impairment, we found a strong inverse relationship between the GDS score and the ratio of neurogranin/BACE1, neurogranin/A $\beta(1-$ 42), and BACE1/t-tau. This indicates that the ratios of biomarkers, especially those including a synaptic biomarker, could add value by identifying patients at risk of persistent cognitive impairment, and by helping to identify signs of AD-related neurodegeneration in its earliest stages. This will have to be verified in long-term follow-up studies, where a particularly interesting group to observe will be patients who can be diagnosed with MDD but who do not yet exhibit any of the typical pathophysiological signs of AD.

The weak correlation of $t$-tau, neurogranin, and $A \beta(1-42)$ with the extent of depression as measured by the GDS suggests that there is no direct pathophysiological link between AD-related pathology and depression. For instance, low A $(1-42)$ levels, a hallmark of AD pathology, were associated with low GDS scores; thus they are not indicative of MDD. Additional evidence that, in general, depression and AD arise from distinct neuropathological pathways comes from Kramberger et al. [6]. These authors demonstrated that t-tau levels are lower in $\mathrm{AD}$ patients with comorbid depression than in other AD patients. They additionally found a negative correlation between CSF t-tau values and depression. Likewise, high 
$A \beta(1-42)$ levels were found in depressed patients compared to a nondepressed group in another study [9]. In our study, these associations were also rather weak, which may explain why other reports failed to find the same correlations with CSF t-tau, A $\beta(1-42)$, and/or neurogranin in the different diagnostic groups [7, 8, 33]. Yet, because of the overlap of some of the cognitive symptoms between $\mathrm{AD}$ and depression, the question arises as to whether, at some level, the pathophysiological mechanisms responsible for these diseases might interact or overlap - particularly among MDD patients who will later develop AD. One study reported a possible interaction in the hippocampus: the neurofibrillary tangles and plaques observed in AD pathology were more pronounced in AD patients with a lifetime history of MDD [40-42]. The cognitive decline in these patients also seemed to progress more quickly [40], suggesting that depression might contribute to the neurodegenerative processes causing dementia. However, the latter study did not implement GDS scores or another standardized method for rating depression. This might explain the discrepancy between its findings and those from another, medium-scale study, which did not reveal any correlation between GDS score and AD pathology [43]. This again highlights the need for additional studies on brain-derived proteins in the CSF to further investigate the pathophysiological mechanisms of AD and depression.

The limitations of the present study should be addressed. The explorative nature of our study is visible in the limited number of patients per diagnostic group. The CSF of a larger number of patients should be analyzed, and should ideally involve longitudinal sampling. In a recent study on older individuals with depressive symptoms, an obvious pattern emerged in which individuals with high and increasing depressive symptoms exhibited an elevated risk of developing dementia [2]. On the basis of single or ratio-based measurements of established biomarkers such as t-tau and $A \beta(1-42)$, it currently remains difficult to make a prognosis on how a case of $\mathrm{AD}$ will progress. This is most likely due to the weak correlation between tangle and plaque pathology and cognitive impairment. A loss of synaptic proteins, on the other hand, has clearly been linked to cognitive deficits [10-12]. In this regard, neurogranin in CSF has potential as a future biomarker, considered either as single analyte [21,22] or in its ratio with BACE1 [27], as De Vos et al. [27] demonstrated an association between high baseline levels of neurogranin/BACE1 and pronounced future cognitive decline.

In conclusion, physicians and clinicians are faced with a major problem when confronted with patients suffering from symptoms of depression and/or MCI. A person in the early stages of AD stands to benefit greatly from early diagnosis, but there is currently no clear way to disentangle the overlapping symptoms of early AD and MDD. In clinical routine, we currently use the CSF biomarkers tau and $A \beta(1-42)$ in the differential diagnosis of AD. Our study does not suggest that neurogranin and BACE1 may have substantial added value as diagnostic biomarkers. However, the preliminary findings on the correlation between the biomarkers and GDS scores now motivate us to study their potential value as prognostic biomarkers. Therefore, we intend to expand the study to a larger cohort of patients, alongside a cognitively healthy control group. In case neurogranin, BACE1, and/or their ratio indeed discriminate between mere depression with cognitive deficits and early AD, this would be a valuable step towards catching cases early and planning appropriate, quality-improving treatments.

\section{Acknowledgements}

The authors thank all patients and caregivers who participated in the study. They also acknowledge Lien Van den Abbeele for technical assistance. 
Schipke et al.: Neurogranin and BACE1 in CSF as Differential Diagnostic Biomarkers

\section{Statement of Ethics}

The study was approved by the Ethics Committee of Charité - Universitätsmedizin Berlin (ClinicalTrials.gov identifier: NCT02686554). Written informed consent was obtained from all patients participating in the study.

\section{Disclosure Statement}

The authors have no conflicts of interest to disclose.

\section{Funding Sources}

O.P. received funding as part of the DELCODE study of the DZNE (German Center for Neurodegenerative Diseases) and from the BIH (Berlin Institute of Health) (grant CR2a, SP5).

\section{References}

1 Van der Mussele S, Fransen E, Struyfs H, Luyckx J, Mariën P, Saerens J, Somers N, Goeman J, De Deyn PP, Engelborghs S: Depression in mild cognitive impairment is associated with progression to Alzheimer's disease: a longitudinal study. J Alzheimers Dis 2014;42:1239-1250.

2 Kaup AR, Byers AL, Falvey C, Simonsick EM, Satterfield S, Ayonayon HN, Smagula SF, Rubin SM, Yaffe K: Trajectories of depressive symptoms in older adults and risk of dementia. JAMA Psychiatry 2016;73:525-531.

3 Leyhe T, Reynolds CF 3rd, Melcher T, Linnemann C, Klöppel S, Blennow K, Zetterberg H, Dubois B, Lista S, Hampel H: A common challenge in older adults: classification, overlap, and therapy of depression and dementia. Alzheimers Dement 2017;13:59-71.

4 Künig G, Jäger M, Stief V, Kaldune A, Urbaniok F, Endrass J: The impact of the CERAD-NP on diagnosis of cognitive deficiencies in late onset depression and Alzheimer's disease. Int J Geriatr Psychiatry 2006;21:911916.

5 Barth S, Schönknecht P, Pantel J, Schröder J: Mild cognitive impairment and Alzheimer's disease: an investigation of the CERAD-NP test battery (in German). Fortschr Neurol Psychiatr 2005;73:568-576.

6 Kramberger MG, Jelic V, Kåreholt I, Enache D, Eriksdotter Jönhagen M, Winblad B, Aarsland D: Cerebrospinal fluid Alzheimer markers in depressed elderly subjects with and without Alzheimer's disease. Dement Geriatr Cogn Dis Extra 2012;2:48-56.

7 Hertze J, Minthon L, Zetterberg H, Vanmechelen E, Blennow K, Hansson O: Evaluation of CSF biomarkers as predictors of Alzheimer's disease: a clinical follow-up study of 4.7 years. J Alzheimers Dis 2010;21:11191128.

8 Pomara N, Bruno D, Sarreal AS, Hernando RT, Nierenberg J, Petkova E, Sidtis JJ, Wisniewski TM, Mehta PD, Pratico D, Zetterberg H, Blennow K: Lower CSF amyloid beta peptides and higher F2-isoprostanes in cognitively intact elderly individuals with major depressive disorder. Am J Psychiatry 2012;169:523-530.

9 Gudmundsson P, Skoog I, Waern M, Blennow K, Pálsson S, Rosengren L, Gustafson D: The relationship between cerebrospinal fluid biomarkers and depression in elderly women. Am J Geriatr Psychiatry 2007;15:832-838.

10 Reddy PH, Mani G, Park BS, Jacques J, Murdoch G, Whetsell W Jr, Kaye J, Manczak M: Differential loss of synaptic proteins in Alzheimer's disease: implications for synaptic dysfunction. J Alzheimers Dis 2005;7:103-117; discussion 173-180.

11 Selkoe DJ: Alzheimer's disease is a synaptic failure. Science 2002;298:789-791.

12 Terry RD, Masliah E, Salmon DP, Butters N, DeTeresa R, Hill R, Hansen LA, Katzman R: Physical basis of cognitive alterations in Alzheimer's disease: synapse loss is the major correlate of cognitive impairment. Ann Neurol 1991;30:572-580.

13 Watson JB, Sutcliffe JG, Fisher RS: Localization of the protein kinase C phosphorylation/calmodulin-binding substrate RC3 in dendritic spines of neostriatal neurons. Proc Natl Acad Sci USA 1992;89:8581-8585.

14 Zhong L, Cherry T, Bies CE, Florence MA, Gerges NZ: Neurogranin enhances synaptic strength through its interaction with calmodulin. EMBO J 2009;28:3027-3039.

15 Díez-Guerra FJ: Neurogranin, a link between calcium/calmodulin and protein kinase C signaling in synaptic plasticity. IUBMB Life 2010;62:597-606.

16 Davidsson P, Blennow K: Neurochemical dissection of synaptic pathology in Alzheimer's disease. Int Psychogeriatr 1998;10:11-23. 
17 Thorsell A, Bjerke M, Gobom J, Brunhage E, Vanmechelen E, Andreasen N, Hansson O, Minthon L, Zetterberg $\mathrm{H}$, Blennow K: Neurogranin in cerebrospinal fluid as a marker of synaptic degeneration in Alzheimer's disease. Brain Res 2010;1362:13-22.

18 De Vos A, Jacobs D, Struyfs H, Fransen E, Andersson K, Portelius E, Andreasson U, De Surgeloose D, Hernalsteen D, Sleegers K, Robberecht C, Van Broeckhoven C, Zetterberg H, Blennow K, Engelborghs S, Vanmechelen E: C-terminal neurogranin is increased in cerebrospinal fluid but unchanged in plasma in Alzheimer's disease. Alzheimers Dement 2015;11:1461-1469.

19 Kester MI, Teunissen CE, Crimmins DL, Herries EM, Ladenson JH, Scheltens P, van der Flier WM, Morris JC, Holtzman DM, Fagan AM: Neurogranin as a cerebrospinal fluid biomarker for synaptic loss in symptomatic Alzheimer disease. JAMA Neurol 2015;72:1275-1280.

20 Janelidze S, Hertze J, Zetterberg H, Landqvist Waldö M, Santillo A, Blennow K, Hansson O: Cerebrospinal fluid neurogranin and YKL-40 as biomarkers of Alzheimer's disease. Ann Clin Transl Neurol 2016;3:12-20.

21 Kvartsberg H, Duits FH, Ingelsson M, Andreasen N, Öhrfelt A, Andersson K, Brinkmalm G, Lannfelt L, Minthon L, Hansson O, Andreasson U, Teunissen CE, Scheltens P, Van der Flier WM, Zetterberg H, Portelius E, Blennow K: Cerebrospinal fluid levels of the synaptic protein neurogranin correlates with cognitive decline in prodromal Alzheimer's disease. Alzheimers Dement 2015;11:1180-1190.

22 Portelius E, Zetterberg H, Skillbäck T, Törnqvist U, Andreasson U, Trojanowski JQ, Weiner MW, Shaw LM, Mattsson N, Blennow K; Alzheimer's Disease Neuroimaging Initiative: Cerebrospinal fluid neurogranin: relation to cognition and neurodegeneration in Alzheimer's disease. Brain 2015;138(pt 11):3373-3385.

23 Lista S, Toschi N, Baldacci F, Zetterberg H, Blennow K, Kilimann I, Teipel SJ, Cavedo E, Dos Santos AM, Epelbaum S, Lamari F, Dubois B, Nisticò R, Floris R, Garaci F, Hampel H; Alzheimer Precision Medicine Initiative (APMI): Cerebrospinal fluid neurogranin as a biomarker of neurodegenerative diseases: a cross-sectional study. J Alzheimers Dis 2017;59:1327-1334.

24 Wellington H, Paterson RW, Portelius E, Törnqvist U, Magdalinou N, Fox NC, Blennow K, Schott JM, Zetterberg $\mathrm{H}$ : Increased CSF neurogranin concentration is specific to Alzheimer disease. Neurology 2016;86:829-835.

25 Kamenetz F, Tomita T, Hsieh H, Seabrook G, Borchelt D, Iwatsubo T, Sisodia S, Malinow R: APP processing and synaptic function. Neuron 2003;37:925-937.

26 Kandalepas PC, Sadleir KR, Eimer WA, Zhao J, Nicholson DA, Vassar R: The Alzheimer's $\beta$-secretase BACE1 localizes to normal presynaptic terminals and to dystrophic presynaptic terminals surrounding amyloid plaques. Acta Neuropathol 2013;126:329-352.

27 De Vos A, Struyfs H, Jacobs D, Fransen E, Klewansky T, De Roeck E, Robberecht C, Van Broeckhoven C, Duyckaerts C, Engelborghs S, Vanmechelen E: The cerebrospinal fluid neurogranin/BACE1 ratio is a potential correlate of cognitive decline in Alzheimer's disease. J Alzheimers Dis 2016;53:1523-1538.

28 Barao S, Zhou L, Adamczuk K, Vanhoutvin T, van Leuven F, Demedts D, Vijverman AC, Bossuyt X, Vandenberghe R, De Strooper B: BACE1 levels correlate with phospho-tau levels in human cerebrospinal fluid. Curr Alzheimer Res 2013;10:671-678.

29 Rosén C, Andreasson U, Mattsson N, Marcusson J, Minthon L, Andreasen N, Blennow K, Zetterberg H: Cerebrospinal fluid profiles of amyloid beta-related biomarkers in Alzheimer's disease. Neuromolecular Med 2012; 14:65-73.

30 Pera M, Alcolea D, Sánchez-Valle R, Guardia-Laguarta C, Colom-Cadena M, Badiola N, Suárez-Calvet M, Lladó A, Barrera-Ocampo AA, Sepulveda-Falla D, Blesa R, Molinuevo JL, Clarimón J, Ferrer I, Gelpi E, Lleó A: Distinct patterns of APP processing in the CNS in autosomal-dominant and sporadic Alzheimer disease. Acta Neuropathol 2013;125:201-213.

31 Kang HJ, Voleti B, Hajszan T, Rajkowska G, Stockmeier CA, Licznerski P, Lepack A, Majik MS, Jeong LS, Banasr $\mathrm{M}$, Son H, Duman RS: Decreased expression of synapse-related genes and loss of synapses in major depressive disorder. Nat Med 2012;18:1413-1417.

32 Rial D, Lemos C, Pinheiro H, Duarte JM, Gonçalves FQ, Real JI, Prediger RD, Gonçalves N, Gomes CA, Canas PM, Agostinho P, Cunha RA: Depression as a glial-based synaptic dysfunction. Front Cell Neurosci 2016; 9:521.

33 Sanfilippo C, Forlenza O, Zetterberg H, Blennow K: Increased neurogranin concentrations in cerebrospinal fluid of Alzheimer's disease and in mild cognitive impairment due to AD. J Neural Transm (Vienna) 2016;123: 1443-1447.

34 Morris JC, Heyman A, Mohs RC, Hughes JP, van Belle G, Fillenbaum G, Mellits ED, Clark C: The Consortium to Establish a Registry for Alzheimer's Disease (CERAD). Part I. Clinical and neuropsychological assessment of Alzheimer's disease. Neurology 1989;39:1159-1165.

35 Welsh KA, Butters N, Hughes JP, Mohs RC, Heyman A: Detection and staging of dementia in Alzheimer's disease. Use of the neuropsychological measures developed for the Consortium to Establish a Registry for Alzheimer's Disease. Arch Neurol 1992;49:448-452.

36 Yesavage JA, Brink TL, Rose TL, Lum O, Huang V, Adey M, Leirer VO: Development and validation of a geriatric depression screening scale: a preliminary report. J Psychiatr Res 1982;17:37-49.

37 Yesavage JA: Geriatric Depression Scale. Psychopharmacol Bull 1988;24:709-711.

38 Schipke CG, Prokop S, Heppner FL, Heuser I, Peters O: Comparison of immunosorbent assays for the quantification of biomarkers for Alzheimer's disease in human cerebrospinal fluid. Dement Geriatr Cogn Disord 2011; 31:139-145 
39 Jack CR Jr, Knopman DS, Jagust WJ, Petersen RC, Weiner MW, Aisen PS, Shaw LM, Vemuri P, Wiste HJ, Weigand SD, Lesnick TG, Pankratz VS, Donohue MC, Trojanowski JQ: Tracking pathophysiological processes in Alzheimer's disease: an updated hypothetical model of dynamic biomarkers. Lancet Neurol 2013;12:207-216.

40 Rapp MA, Schnaider-Beeri M, Grossman HT, Sano M, Perl DP, Purohit DP, Gorman JM, Haroutunian V: Increased hippocampal plaques and tangles in patients with Alzheimer disease with a lifetime history of major depression. Arch Gen Psychiatry 2006;63:161-167.

41 Rapp MA, Schnaider-Beeri M, Purohit DP, Perl DP, Haroutunian V, Sano M: Increased neurofibrillary tangles in patients with Alzheimer disease with comorbid depression. Am J Geriatr Psychiatry 2008;16:168-174.

42 Tsopelas C, Stewart R, Savva GM, Brayne C, Ince P, Thomas A, Matthews FE; Medical Research Council Cognitive Function and Ageing Study: Neuropathological correlates of late-life depression in older people. Br J Psychiatry 2011;198:109-114.

43 McCutcheon ST, Han D, Troncoso J, Koliatsos VE, Albert M, Lyketsos CG, Leoutsakos JS: Clinicopathological correlates of depression in early Alzheimer's disease in the NACC. Int J Geriatr Psychiatry 2016;31:13011311. 